\title{
Vitamin B12 Deficit Status among Type 2 Diabetes Mellitus Patients - A Review
}

\author{
Lata Kanyal Butolaํ, Roshan Kumar Jha ${ }^{2}$, Ranjit Ambad ${ }^{3}$, Deepika Kanyal' ${ }^{4}$, Jayshri Jankar ${ }^{5}$
}

1, 2, 3, 5 Department of Biochemistry, Jawaharlal Nehru Medical College, Sawangi, Wardha, Maharashtra, India.

${ }^{4}$ Department of Hospital Administration, Jawaharlal Nehru Medical College, Sawangi, Wardha, Maharashtra, India.

\section{ABSTRACT}

Several studies have confirmed the increased incidence of vitamin B12 deficiency in patients with type 2 diabetes mellitus. Metformin is currently the most commonly used anti-diabetic drug, metformin being prescribed as first-line therapy for patients with type 2 diabetes mellitus (T2DM) worldwide. Other disorders including insulin resistance, such as polycystic ovary syndrome (PCOS), can also be treated with metformin.

Metformin has positive effects on metabolism, weight loss, and vascular defence of carbohydrates, but it also has significant side effects. Patients on long-term treatment with metformin, for example, have been shown to be at risk of anemia. This may be because of a decrease in metformin-related vitamin B12. It is estimated that 30 percent of patients undergoing long-term metformin treatment have experienced vitamin B12 malabsorption, with a 14 percent to 30 percent reduction in serum vitamin B12 concentration.

A critical nutrient for wellbeing is vitamin B12. It plays a significant role in the functioning and the production of red blood cells in the brain and nervous system. In addition to anemia, a deficiency of vitamin B12 may increase the severity of peripheral neuropathy in T2DM patients. In addition, since vitamin B12 is involved in the most critical homocysteine (Hcy) metabolism pathway, a decrease in vitamin B12 will increase plasma concentrations of Hcy, which in patients with T2DM and PCOS is closely linked to cardiovascular disease.

Evaluating serum vitamin B12 levels will also provide an early diagnosis of the status of the deficiency. This will offer an incentive for harm caused by routine screening and early detection to be reversed.

\section{KEY WORDS}

Vitamin B12, Metformin, Diabetes Mellitus, Glycated Hb
Corresponding Author: Dr. Deepika Kanyal, Department of Hospital Administration, Jawaharlal Nehru Medical College, Sawangi, Wardha, Maharashtra, India. E-mail: Kanyallatat1010@gmail.com

DOI: $10.14260 /$ jemds/2021/370

How to Cite This Article: Butola LK, Jha RK, Ambad R, et al. Vitamin $B 12$ deficit status among type 2 diabetes mellitus patients - a review. J Evolution Med Dent Sci 2021;10(23):1794-1798, DOI: 10.14260/jemds/2021/370

Submission 29-10-2020,

Peer Review 07-04-2021,

Acceptance 15-04-2021,

Published 07-06-2021.

Copyright (C) 2021 Lata Kanyal Butola et al. This is an open access article distributed under Creative Commons Attribution License [Attribution 4.0 International (CC $B Y 4.0)]$ 


\section{BACKGROUND}

Metformin is commonly used as a first-line drug inpatients with insulin. It is known as an insulin sensitiser because it helps to lower insulin levels, decreases the production of hepatic glucose, increases the use of peripheral glucose, and helps to consume and use intestinal glucose. It triggers adenosine monophosphate (AMP)- dependent protein kinase and enters the cell via organic cation transporters. ${ }^{1,2}$ Metformin's key site of action is the liver where it suppresses the development of glucose and promotes tissue glucose absorption via glucose transporters and inhibits hepatic gluconeogenesis by mediating liver kinase B 1 (LKB1) activation, thus, promoting gluconeogenesis enzyme synthesis. It contributes to hyperglycemia by suppressing the liver's production of glucose.

Metformin was approved by the Food and Drug Administration (FDA) in 1994 and was approved in America in 1996 when it was shown to decrease mortality and decrease diabetes-related complications in patients with type 2 obese diabetes. It is estimated that metformin is regularly administered to 120 million diabetic patients around the world. The World Health Organization (WHO) study in 1985 stated the usefulness of $\mathrm{HbA1c}$ for diabetes. HbA1c was recommended by American Diabetes Association (ADA) and the world's major expert committee and association for the diagnosis of type 2 diabetes mellitus by 2010. Diabetes Management and Implications Trial and significance on T2DM was provided by the United Kingdom Prospective Diabetes Study in 2010 by the ADA-Organized International Expert Committee and recommended the adoption of HbA1c for the diagnosis of diabetes at a cut-off of 6.5 percent clinical significance of $\mathrm{HbA1c}$ in T1DM. In the routine treatment of patients with T1DM and T2DM tests of HbA1C and blood glucose levels are used. ${ }^{3-5}$

\section{EFFECT OF METFORMIN ON VITAMIN B12 LEVELS}

Use of metformin interestingly leads to deficiency of vitamin B12 which can cause neuropathy, ranging from paraesthesia and diminished peripheral sensation to derangement of mental status. There is need of assessing vitamin B12 in individuals with T2DM who are on medication to metformin. 6,7 Metformin is used to treat diabetes, affects the receptors of calcium-dependent ileal cell membrane necessary for intrinsic factor B12 uptake and decreases up to 30 percent serum vitamin B12 absorption.

Deficiency of vitamin B12 induced by metformin intake induces altered small intestine motility, bacterial flora, causes inhibition and inactivates vitamin B12 absorption. As it interacts with endocytic cubulin receptors or the influence of calcium on the cell membrane, it modifies the levels of intrinsic factors. It was proposed to play a role as a protonated biguanide group that prevents calcium-dependent absorption reserved for calcium supplementation. ${ }^{3}$

T2DM is a multifactorial illness associated with the metabolism of energy, specifically carbohydrates and fats, leading to macrovascular and microvascular complications most of the time. Deficiency of red blood cells (RBC) vitamin
B12 in patients contains reduced glutathione or enzymes necessary for degradation of ribose glucose and further contributes to the elevation of coenzyme $\mathrm{A}$ in the liver. ${ }^{8}$ Increased metabolism of fatty acids leads to elevation of intramitochondrial acetyl coenzyme A, which inhibits pyruvate dehydrogenase, leading to inhibition of phosphofructokinase.

Advanced glycation end products (AGE) are developed due to hyperglycaemia, which develops to pathogenesis of demyelination by macrophages. Abnormalities in Schwann cell includes reactive as well as degenerative changes which leads to neuropathy followed by initial demyelination, axonal degeneration and nerve fibre loss induction. In patients with T2DM, monitoring of vitamin B12 is important as a decrease in its levels hampers the maintenance of the enzyme mechanism necessary for the use of carbohydrates and fats. ${ }^{9}$

The study conducted by Monique Nervo et al. to determine the prevalence of vitamin B12 deficiency and serum vitamin B12 related factors in a group of metformin-treated Brazilian diabetic patients. In 10 patients (6.9 percent), serum vitamin B12 levels were low ( $<125 \mathrm{pmol} / \mathrm{l})$ and possibly low (125 pmol / l to $250 \mathrm{pmol} / \mathrm{l}$ ) in 53 patients (36.8 percent) and were associated with age, estimated vitamin B12 intake and body weight. The association between the dosage of metformin and the duration of metformin administration with serum concentrations of vitamin B12 was $r=-0.114, P=0.173$ and $r$ $=-0.128, \mathrm{P}=0.129$. There was a sufficient daily intake of vitamin B12 (> 2.4 $\mu \mathrm{g}$ ) in 68 patients (47 percent). The study concluded that serum vitamin B12 levels in $6.9 \%$ and $36.8 \%$ of 144 diabetic patients treated with metformin for more than one year were low and likely low, respectively. The incidence of deficiency of vitamin B12 varies according to the amount of serum vitamin B12 cutoff used 10,12. Ideally, in patients with potentially low serum vitamin B12 levels, serum methylmalonic acid should be tested. ${ }^{10}$

The mean prevalence of vitamin B12 concentrations $<150$ pml / l was 9.7 percent (95 percent CI 6.6 - 13.7 percent) in the total cohort $(\mathrm{N}=298)$ in a cross-sectional study. In type 2 diabetes patients not taking metformin $(\mathrm{N}=134)$, the prevalence was 4.4 percent (95 percent CI 1.6 - 9.4 percent) compared to 14.1 percent in metformin users $(\mathrm{N}=164)(95$ percent $\mathrm{CI} 9.2$ - 20.4 percent; $\mathrm{P}=0.006)$. The metformin dose increased per $100 \mathrm{mg}$ step $(\mathrm{OR}=1.081, \mathrm{P}=0.014)$, while the use of proton pump inhibitors (PPI) decreased the risk of vitamin $\mathrm{B} 12$ deficiency in logistic regression $(\mathrm{OR}=0.322, \mathrm{P}=$ 0.037). However, the use of metformin did not predict anaemia or neuropathy risk. In patients with type 2 diabetes who use metformin, the incidence of vitamin B12 deficiency is higher than that of patients who do not use metformin. However, the use of metformin failed to predict the risk of developing anaemia or neuropathy. ${ }^{11}$

Study conducted in Korea shows that vitamin B12 deficiency in metformin-treated T2DM patients was $9.5 \%$ (N = 76) and mean vitamin B12 level $(662.5 \pm 246.7 \mathrm{pg} / \mathrm{ml})$. Long-term and high-dose vitamin B12 deficient patients were on treatment compared to non-deficient patients, and the discrepancies were substantial $(\mathrm{P}<0.001)$. The modified odds ratio for 1000 - $2000 \mathrm{mg}$ was about 2.52 relative to a daily metformin dose of $<1000 \mathrm{mg}$ (955CI, $1.27-4.99, \mathrm{P}=.0080$ ) and 3.80 (95 percent $\mathrm{CI}, 1.82-7.92, \mathrm{P}<0.001$ ). Their research concluded that patients with type 2 diabetes should be tested for vitamin B12 with treatment. Specifically patients on 
medication especially at higher doses of metformin for long duration. ${ }^{10}$

Study conducted in Nigeria observed there was vitamin B12 deficiency in $8.6 \%$ and borderline deficiency in $26.0 \%$ of the patients who have been using metformin. Its level was significantly decreased in patients on medication for more than and equal to 10 years as compared to patients with medication on less than 10 years.

Similarly, those who were on metformin dose of more than $1000 \mathrm{mg}$ per day had significantly lower level of Vitamin B12 in patients with T2DM as compared to those who were on low dose of metformin. ${ }^{12}$

A cross sectional study was conducted by Sujatha Rani Akuri (2014) in Hyderabad, India, in which 89 patients both male and female of $35-70$ years age group with type 2 diabetes mellitus and 89 age matched healthy control group was included.

There is a strong difference between case and control mean values of $214.63 \mathrm{pg} / \mathrm{ml}$ and $693.39 \mathrm{pg} / \mathrm{ml}$, and a substantial difference between case and control mean values of $\mathrm{P}<0.001$ has been found. It was concluded from this analysis that there is a clear association between low and borderline vitamin B12 levels and diabetic neuropathy, i.e. $\mathrm{P}<$ 0.001. Compared to healthy people, type 2 diabetic patients on metformin therapy and vegetarians have low vitamin B12 levels, which is known as a comorbid factor contributing to diabetic neuropathy and diabetic foot. ${ }^{13}$

A cross-sectional analysis conducted in Hubli, Karnataka, by Anand Kalakappa Koppad et al. (2017). Total 100 Type 2 diabetes mellitus. Most patients with a mean diabetes mellitus (DM) period of 4.8 years were in the 45 - 59 year age range. In the age group $>60$ years, 42 percent of the overall vitamin B12 deficiency was predominantly seen; males were 54.5 percent more deficient. Most patients with vitamin B12 deficiency have a diabetes period of $>10$ years and most of them have been taking metformin for $>10$ years and a higher dose of $>2$ $\mathrm{g} /$ day. None of the diabetes patients with normal body mass index (BMI) are deficient in vitamin B12. Vitamin B12 deficiency is greater in 52.5 percent of overweight patients and 51.3 percent of obese patients. The current study concludes that the risk of developing vitamin B12 deficiency is increased by age and longer duration of diabetes and that the duration of diabetes is associated with B12 deficiency. There are elevated levels of fasting blood sugar (FBS), post prandial blood sugar (PPBS), and HbA1c in vitamin B12 deficient T2DM patients on longer metformin therapy. ${ }^{14}$

A cross sectional study conducted by Shamin Ahmed et al. (2016) in Guwahati India, 80 patients with diagnosed T2DM and 40 healthy controls were taken. The age distribution in cases was $52.15 \pm 8.66$ and $54.14 \pm 7.44$ in controls. In cases $40 \%$ were females and $60 \%$ were males and in control group $30 \%$ were females and $70 \%$ were males. Mean vitamin B12 in controls was 597.47pg / $\mathrm{ml}$ and 404.36pg / $\mathrm{ml}$ in cases which is significantly low in cases, $\mathrm{P}<0.0001$.

In cases, prevalence of vitamin B12 deficiency was $10 \%$ and borderline deficiency was $17.5 \%$. A significant correlation with vitamin B12 deficiency was seen between increasing age and duration of diabetes, $\mathrm{P}<0.05$. Mean fasting plasma glucose (FPG) in controls was $90.68 \mathrm{mg} / \mathrm{dl}$ and in cases was 204.15 $\mathrm{mg} / \mathrm{dl}$ which was extremely significant, $\mathrm{P}<0.0001$. Mean $\mathrm{HbA} 1 \mathrm{c}$ in cases was $8.49 \%$ and in controls was $5.1 \%$ which is significant, $\mathrm{P}<0.0001$. From the present study it was concluded that there is a decrease serum vitamin B12 level with the increase in $\mathrm{HbA1c}$, cholesterol and triglyceride levels. ${ }^{15}$

A cross sectional study was conducted by Ashima Badyal et al. in Jammu (2018) including 50 patients diagnosed with type 2 DM in the age group of $40-80$ year and 50 genders matched healthy controls. The mean age of controls was (53.1 $\pm 8.2)$ and cases was $(55.0 \pm 6.8)$. In cases $40 \%$ were females and $60 \%$ were males and in control group $33 \%$ females and $67 \%$ males. The mean vitamin B12 in cases was $353 \mathrm{pg} / \mathrm{ml}$ and control was $602 \mathrm{pg} / \mathrm{ml}$ which was low in cases. The mean FPG in cases and control was 211.61 and $93.17 \mathrm{mg} / \mathrm{dl}$ and mean HbA1c was 8.94 in cases and 5.21 in controls it was significantly higher in cases while vitamin B12 deficiency was associated with increasing age and increased duration of diabetes. From the present study it is concluded that prevention and management of diabetes is a major health issue, vitamin B12 deficiency leads to accumulation of serum homocysteine which causes significant complications and increased morbidity. ${ }^{16}$

A cross sectional study was conducted by Vivek kumar et al. in Uttar Pradesh (2017) in which total 93 type 2 diabetic patients were included and mean age was 51.24 years with minimum age of 39 years and maximum age was 60 years; out of which 51 patients were males and 42 were females. 70 patients were non-vegetarian and 23 patients were vegetarian and minimum dose of metformin was 5 years and the maximum was 16 years in duration of 9.49 years. In the present study vitamin B12 deficiency was found in 38 patients out of whom 25 were non-vegetarians and 13 were vegetarian. The present study concluded that vitamin B12 deficiency is common in diabetic patients on prolonged metformin based oral hypoglycaemic agents (OHA) therapy. Vegetarian population is more deficient than non-vegetarians, but the difference is not statistically significant which is in favor of metformin associated vitamin B12 deficiency in nonvegetarian population. ${ }^{17}$

A cross sectional survey was conducted by Jaya Prasad Tripathy et al. in Punjab (2017) to see the prevalence and risk factor of diabetes in Punjab, in a multistage stratified sample of 5127 individuals. 2499 participants were assayed for blood parameters. The prevalence of DM among the study was $8.3 \%$, whereas prevalence of pre-diabetes was $6.3 \%$; out of all persons $18 \%$ were known case of DM. The study reported that there was a high prevalence of diabetes in undiagnosed cases in the adult population, with uncontrolled blood sugar levels, there should be systematic screening and awareness program to identify undiagnosed cases in community. ${ }^{18}$

A prospective case control study was conducted by Daryl et al. in Calgary, Canada (2010). 226 patients with type 2 diabetes mellitus and peripheral neuropathy were included out of whom 104 patients were excluded and 122 patients were eligible for analysis, among them 59 patients received metformin therapy for $>6$ months and the remaining patients were not on metformin. From the present study it was concluded that patients with T2DM, peripheral neuropathy and patients on metformin have lower serum cobalamin, higher serum homocysteine and methylmalonic acid. Metformin exposure leads to peripheral neuropathy in T2DM patients and screening for cobalamin deficiency and systemic cobalamin therapy should be done. ${ }^{19}$ 
A cross sectional study was conducted by Matthew C. Pflipsen et al. in Huachuca (2009) on 203 T2DM patients. 8 patients were excluded and the remaining 195 patients were enrolled. Vitamin B12 levels ranged from 91 to $2818 \mathrm{pg} / \mathrm{ml}$, only one individual had B12 level $<100 \mathrm{pg} / \mathrm{ml}, 79$ patients had levels between $100-350 \mathrm{pg} / \mathrm{ml}, 43$ patients between of these 79 patients had elevated homocysteine or methylmalonic acid, resulting in total $22 \%$ of patients diagnosed with metabolic vitamin B12 deficiency. They compared between the patients with and without B12 deficiency. Metformin use was not associated with vitamin B12 deficiency. This study concluded that primary care physicians recognised that one fifth of diabetic patients may have vitamin B12 deficiency, diagnosis of vitamin B12 will manage the comorbidities of diabetes, mainly neuropathy. Treatment with multivitamin should be given to reduce the risk of vitamin B12 deficiency in type 2 diabetes mellitus. ${ }^{20}$

A descriptive-comparative study was conducted in 400 types 2 DM patients. There were two classes of enrolled patients, those receiving metformin 6 months earlier $(\mathrm{N}=200)$ and those receiving hypoglycemic agents other than metformin ( $\mathrm{N}=200)$. Vitamin B12 levels were substantially lower in patients receiving metformin $(320.94 \pm 141.34$ vs $408.50 \pm 175.07 \mathrm{pmol} / \mathrm{l}, \mathrm{P}<0.001$ ) and the incidence of vitamin B12 deficiency was higher (14.5 vs 2 percent, $\mathrm{P}<$ $0.001)$. There was a negative correlation between weight $(\mathrm{r}=$ - 0.18, $\mathrm{P}<0.001)$ and BMI $(\mathrm{r}=-0.11, \mathrm{P}=0.02)$ levels of vitamin $\mathrm{B} 12$ and a positive correlation between vitamin B12 and metformin administration $(\mathrm{r}=0.26, \mathrm{P}<0.001)$. And their study concluded that those patients who had been on treatment for a long period of time were vitamin B12 deficient relative to non-metformin patients. This correlation of serum vitamin B12 deficiency with metformin therapy prescribes periodic measurements of serum vitamin B12 in patients receiving metformin for long-term use. ${ }^{21}$

\section{DISCUSSION}

Metformin is one of the most commonly used drugs for T2DM the world over and the period of treatment has been increased which increases the prevalence of metformin induced vitamin B 12 deficiency. Vitamin B12 is recommended for monitoring the clinical significance of vitamin B12 deficiency associated with metformin therapy in patients with T2DM, especially those on metformin medication. Gastric parietal cell produces intrinsic factors which are required for vitamin B12 absorption from the gastrointestinal tract (GIT). Metformin treatment results in deficiency of vitamin B12, mediated by depression of intrinsic factor secretion.

Clinical deficiency of vitamin B12 may progress to altered mental status, megaloblastic anaemia and neuronal damage. That is the reason, peripheral neuropathy caused due to vitamin B12 deficiency and same due to diabetes is confused and may contribute to aggravation of diabetic peripheral neuropathy which may lead to permanent neurological damage. This is a fact that due to clinical benefits of metformin its associated side effects are generally not taken seriously and investigation is very rare.

Vitamin B12 deficiency must be taken seriously especially in elderly people, vegans, in patients with intestinal disorders, as well as inpatients with autoimmune disorders such as graves' disease, thyroiditis, vitiligo, and in patients receiving proton pump inhibitor, histamine receptor antagonists or biguanides for prolonged period.

\section{CONCLUSIONS}

Patients on metformin therapy should routinely check holotranscobalamin, methylmalonic acid and homocysteine levels. Thus, evaluating vitamin B12 levels in serum would provide early diagnosis of the deficiency status. This would provide an opportunity for the reversal of damage caused by routine screening and early diagnosis.

Financial or other competing interests: None.

Disclosure forms provided by the authors are available with the full text of this article at jemds.com.

\section{REFERENCES}

[1] Ralston S, Penman I, Strachan M, et al. Hepatalogy. In: Davidson's Principles and Practice of Medicine. 23 $3^{\text {rd }}$ edn. Elsevier Publication 2018: p. 882-3.

[2] Kasper D, Fauci A, Hauser S, et al. Diabetes mellitus. Harrison's Principles of Internal Medicine. 19th edn. New York: McGraw-Hill 2015: p. 2422, 2399.

[3] Kanyal L, Madhura TK, Mujawar A. Status of vitamin b12 in type 2 diabetes mellitus patients taking metformin based oral hypoglycemic agent-a cross sectional study. Indian Journal of Basic and Applied Medical Research 2019;9(1):18-26.

[4] Madhura TK, Kanyal L, Mujawar A. Effect of glycemic control on vitamin B12 status in type 2 diabetes mellitus. Indian Journal of Basic and Applied Medical Research 2019;1(9):7-17.

[5] Butola LK, Meshram A, Dhok A, et al. Urticaria due to adverse drug reaction in diabetes: a case presentation. J Evolution Med Dent Sci 2020;9(37):2764-6.

[6] Kim J, Ahn CW, Fang S, et al. Association between metformin dose and vitamin B12 deficiency in patients with type 2 diabetes. Medicine (Baltimore) 2019;98(46):e17918.

[7] Butola LK, Kute PK, Anjankar A, et al. Vitamin B12-do you know everything? J Evolution Med Dent Sci 2020;9(42):3139-46.

[8] Rusher DR, Pawlak R. A review of 89 published case studies of vitamin B12 deficiency. J Hum Nutr Food Sci 2013;1(2):1008.

[9] Kibirige D, Mwebaze R. Vitamin B12 deficiency among patients with diabetes mellitus: is routine screening and supplementation justified? J Diabetes Metab Disord 2013;12(1):17.

[10] Nervo M, Lubini A, Raimundo FV, et al. Vitamin B12 in metformin - treated diabetic patients: a cross - sectional study in Brazil. Rev Assoc Med Bras 2011;57(1):46-9.

[11] De Groot-Kamphuis DM, Van Dijk PR, Groenier KH, et al. Vitamin B12 deficiency and the lack of its consequences in type 2 diabetes patients using metformin. Neth J Med 2013;71(7):386-90. 
[12] Akinlade KS, Agbebaku So, Rahamon SK, et al. Vitamin B12 levels in patients with type 2 diabetes mellitus on metformin. Ann Ib Postgrad Med 2015;13(2):79-83.

[13] Akuri RS. Significance of Vitamin B12 deficiency in patients with type 2 diabetes mellitus. International Journal of Pharma and Bio Science 2014;5(3):647-52.

[14] Koppad AK, Patil G, Baligar BD, et al. Evaluation of serum vitamin b12 levels in type 2 diabetes patients on metformin therapy attending a tertiary care hospital. Journal of Evidence Based Medicine and Healthcare 2017;4(90):5399-404.

[15] Ahmed S, Rohman S. Study of serum Vitamin B12 and its correlation with lipid profile in type 2 diabetes mellitus. Indian Journal of Basic and Applied Medical Research 2016;5(4):92-103.

[16] Badyal A, Kumar S. Role of Vitamin B12 deficiency in patients with type 2 diabetes mellitus. Journal of Medical Science and Clinical Research 2018;6(2):235-8.

[17] Verma VK, Nim RK, Singh PS, et al. Vitamin B12 deficiency among vegetarian and non-vegetarian diabetic population receiving prolonged metformin based oral hypoglycemic agents therapy. International Journal of Advances in Medicine 2017;4(4):1150-4.

[18] Tripathy JP, Thakur JS, Jeet G, et al. Prevalence and risk factors of diabetes in a large community-based study in North India: results from a STEPS survey in Punjab, India. Diabetol Metab Syndr 2017;9(1):8.

[19] Wile DJ, Toth C. Association of metformin, elevated homocysteine and methylmalonic acid levels and clinically worsened diabetic peripheral neuropathy. Diabetes Care 2010;33(1):156-61.

[20] Pflipsen MC, Oh RC, Saguil A, et al. The prevalence of vitamin B12 deficiency in patients with type 2 diabetes: a cross-sectional study. J Am Board Fam Med 2009;22(5):528-34.

[21] Niafar M, Jamali B, Alikhah H, et al. Vitamin B12 deficiency in type 2 diabetic patients on metformin in 15 th European Congress of Endocrinology. Bio Scientifica 2013;32. 